\title{
The nuclear symmetry energy and the breaking of the isospin symmetry: how do they reconcile with each other?
}

\author{
$X$. Roca-Maza ${ }^{1,2, *}, G$. Colò ${ }^{1,2}$, and $H$. Sagawa ${ }^{3,4}$ \\ ${ }^{1}$ Dipartimento di Fisica, Università degli Studi di Milano, 20133 Milano, Italy. \\ ${ }^{2}$ INFN, Sezione di Milano, 20133 Milano, Italy. \\ ${ }^{3}$ RIKEN Nishina Center, Wako 351-0198, Japan \\ ${ }^{4}$ Center for Mathematics and Physics, University of Aizu, Aizu-Wakamatsu, Fukushima 965-8560, Japan
}

\begin{abstract}
Our current knowledge of the Equation of State of asymmetric nuclear matter around saturation density and of the energy of the Isobaric Analog State in a heavy nucleus such as ${ }^{208} \mathrm{~Pb}$ seem to be in contradiction. In Ref. [1], the problem has been highlighted and a solution has been proposed. In the present contribution, we overview the aforementioned work by giving some new details not previously published.
\end{abstract}

\section{Introduction}

One of the most outstanding problems in nuclear physics is the accurate determination of the nuclear equation of state $(\mathrm{EoS})[2,3]$. The nuclear symmetry energy is one of the fundamental ingredients to describe the EoS when dealing with isospin asymmetric matter $[4,5]$ and its determination may entail profound consequences in our understanding of heavy-ion reactions [6], neutron stars [7], or of the Standard Model via atomic parity violation [8].

Specifically, the symmetry energy is the energy per particle needed to change protons into neutrons in uniform matter at a given density $\rho$. At saturation density of symmetric matter, $\rho_{0} \approx 0.16 \mathrm{fm}^{-3}$, its value is estimated to be between 28-35 MeV [3]. If $\beta$ is the local neutron-proton asymmetry, $\beta \equiv\left(\rho_{n}-\rho_{p}\right) / \rho$, the energy per particle in matter having neutron-proton imbalance is a function $\frac{E}{A}(\rho, \beta)$. Such function can be expanded in even powers of $\beta$ owing to isospin symmetry (the Coulomb force has to be taken out when dealing with a uniform system). By retaining only the quadratic term we can write

$$
\frac{E}{A}(\rho, \beta)=\frac{E}{A}(\rho, \beta=0)+S(\rho) \beta^{2} .
$$

This equation defines the symmetry energy $S(\rho)$, that is, the difference between the energy per particle $E / A$ in neutron and symmetric matter.

It is customary to expand Eq. (1) around saturation density as

$$
S(\rho)=J+L\left(\frac{\rho-\rho_{0}}{3 \rho_{0}}\right)+\frac{1}{2} K_{s y m}\left(\frac{\rho-\rho_{0}}{3 \rho_{0}}\right)^{2}+\ldots,
$$

where different parameters have been defined, namely $J \equiv S\left(\rho_{0}\right), L \equiv 3 \rho_{0} S^{\prime}\left(\rho_{0}\right)$, and $K_{\text {sym }} \equiv 9 \rho_{0}^{2} S^{\prime \prime}\left(\rho_{0}\right)$.

*e-mail: xavier.roca.maza@mi.infn.it
Predictions on these parameters based on different nuclear models has been documented along the years. While $K_{\text {sym }}$ is basically not known, the error on $L$, referred to as the "slope parameter", is believed to be still significantly larger than the error on $J$ : ranges between 30 $90 \mathrm{MeV}$ approximatelly [2, 3, 9-11]. Many authors have pointed out a correlation between $L$ and the neutron skin $\Delta R_{\mathrm{np}} \equiv\left\langle r_{n}^{2}\right\rangle^{1 / 2}-\left\langle r_{p}^{2}\right\rangle^{1 / 2}$ of a heavy nucleus like ${ }^{208} \mathrm{~Pb}[12-$ 15]. Accurate measurements of the neutron skin are still required to pin down the value of $L$ [16-20].

The difficulties in determining the symmetry energy are essentially associated with our incomplete understanding of the strong interaction in the low-energy regime. Therefore, to find a connection with an observable that is not sensitive to the strong force becomes an asset. The Isobaric Analog State (IAS) is one of the well established properties of nuclei that is measured accurately, and is only sensitive to the isospin symmetry breaking (ISB) in the nuclear medium due to Coulomb interaction and, to a lesser extent, the other effects discussed below.

At present, nuclear Energy Density Functionals (EDFs), based on the Density Functional Theory, constitute the only theoretical framework in which the neutron skins and the IAS energies can be consistently calculated from a microscopic perspective, in medium-heavy nuclei [21]. There exist different types of EDFs. In particular, results from the Skyrme and covariant density dependent type of functionals will be presented here. Those are based on the Hartree-Fock (HF) and Hartree approaches, respectively, for the desncription of ground-state properties; and, self-consistently, on the charge-exchange Random Phase Approximation (RPA) for the study of nuclear collective excitations [22, 23].

Within the Skyrme functionals, SAMi [24] has been shown to be specially accurate in the description of chargeexchange resonances. In Refs. [1, 25], it is shown that 


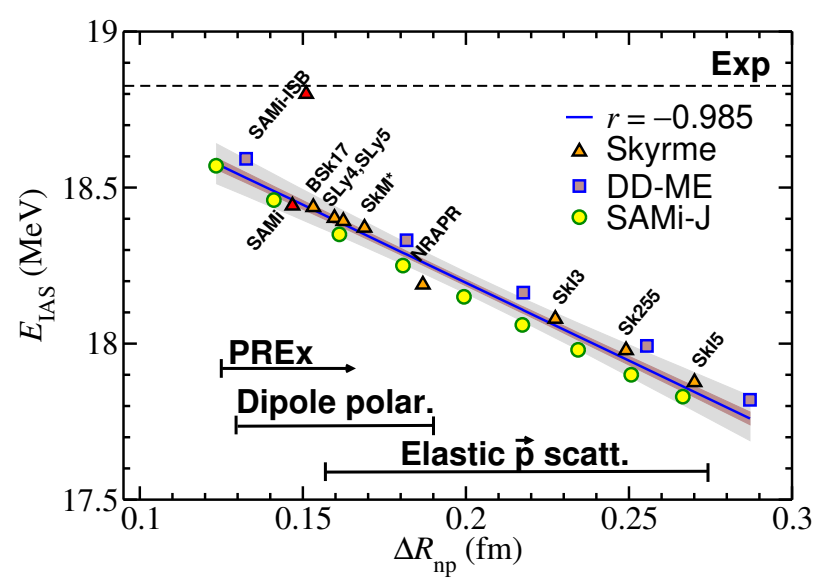

Figure 1. Energy of the IAS as a function of $\Delta R_{n p}$. The arrows indicate the experimental results from polarized proton elastic scattering [29], parity violating elastic electron scattering [19], and from the electric dipole polarizability [30]. Figure is taken from Ref. [1].

families of functionals, based either on SAMi [26] or on the covariant functional DD-ME [27], in which J and L vary systematically, provide predictions for the IAS energy, $E_{\mathrm{IAS}}$, as a function of $\Delta R_{\mathrm{np}}$ in ${ }^{208} \mathrm{~Pb}$ that follow a linear correlation. For the sake of completeness, results associated with other Skyrme interactions are also plotted. Such correlation can be understood in simple physical terms. The excitation energy $E_{\text {IAS }}$ is defined as the energy difference between the analog state $|A\rangle$ and the parent state $|0\rangle$. The parent state is an eigenstate of the Hamiltonian $\mathcal{H}$ with $N$ neutrons and $Z$ protons and the analog state can be defined as $|A\rangle \equiv T_{-}|0\rangle\left\langle 0\left|T_{+} T_{-}\right| 0\right\rangle^{-1 / 2}$ (cf. in Ref. [28]). Hence,

$$
E_{\mathrm{IAS}}=\langle A|\mathcal{H}| A\rangle-\langle 0|\mathcal{H}| 0\rangle=\frac{\left\langle 0\left|T_{+}\left[\mathcal{H}, T_{-}\right]\right| 0\right\rangle}{\left\langle 0\left|T_{+} T_{-}\right| 0\right\rangle}
$$

and assuming good isospin in the parent state $\left\langle 0\left|T_{+} T_{-}\right| 0\right\rangle=$ $N-Z+\left\langle 0\left|T_{-} T_{+}\right| 0\right\rangle=N-Z$,

$$
E_{\mathrm{IAS}}=\frac{1}{N-Z}\left\langle 0\left|\left[T_{+},\left[\mathcal{H}, T_{-}\right]\right]\right| 0\right\rangle .
$$

Due to the structure of Eq. (3), $E_{\text {IAS }}$ depends on isospin breaking parts of the $\mathcal{H}$ only. In nuclear physics, the main isospin breaking term is known to be due to the Coulomb interaction. Therefore, the bulk contribution to Eq. (3) will be due to the difference in the expectation value of the Coulomb matrix elements between proton and neutron distributions. That is, for the direct Coulomb term assuming an independent particle model

$$
E_{\mathrm{IAS}}^{\mathrm{C} \text {,direct }}=\frac{1}{N-Z} \int\left[\rho_{n}(\vec{r})-\rho_{p}(\vec{r})\right] U_{\mathrm{C}}^{\mathrm{direct}}(\vec{r}) d \vec{r},
$$

where $U_{\mathrm{C}}\left(\vec{r}_{1}, \vec{r}_{2}\right)$ is the direct part of the Coulomb energy potential generated by the electric charge distribution $\rho_{\mathrm{ch}}(\vec{r})$,

$$
U_{\mathrm{C}}^{\mathrm{direct}}(\vec{r})=\int \frac{e^{2}}{\left|\vec{r}_{1}-\vec{r}\right|} \rho_{\mathrm{ch}}\left(\vec{r}_{1}\right) d \vec{r}_{1}
$$

The Coulomb exchange contribution can be evaluated in an analogous way but will be much smaller than the Coulomb direct part.

In order to understand in simple terms the relation between the $E_{\mathrm{IAS}}$ and $\Delta R_{\mathrm{np}}$ we will evaluate Eq. (5) within a simple yet physical model. Assuming a uniform neutron and proton distributions of radius $R_{n}$ and $R_{p}$ respectively, one can evaluate the Coulomb energy potential as

$$
U_{\mathrm{C}}^{\text {direct }}(\vec{r})= \begin{cases}\frac{Z e^{2}}{2 R_{p}}\left(3-\frac{r^{2}}{R_{p}^{2}}\right) & \text { for } r<R_{p} \\ \frac{Z e^{2}}{r} & \text { for } r>R_{p}\end{cases}
$$

and, therefore, obtain a simple formula for estimating the bulk part of $E_{\mathrm{IAS}}$ in terms of physical quantities

$$
\begin{aligned}
E_{\mathrm{IAS}} & \approx E_{\mathrm{IAS}}^{\mathrm{C}, \text { direct }} \\
& \approx \frac{6}{5} \frac{Z e^{2}}{R_{p}}\left(1-\frac{1}{2} \frac{N}{N-Z} \frac{R_{n}-R_{p}}{R_{p}}\right) \\
& \approx \frac{6}{5} \frac{Z e^{2}}{r_{0} A^{1 / 3}}\left(1-\sqrt{\frac{5}{12}} \frac{N}{N-Z} \frac{\Delta R_{\mathrm{np}}}{r_{0} A^{1 / 3}}\right),
\end{aligned}
$$

that is, the IAS energy should decrease with increasing neutron skin thickness, $\Delta R_{\mathrm{np}} \equiv \sqrt{3 / 5}\left(R_{n}-R_{p}\right)$. For example, for the case of ${ }^{208} \mathrm{~Pb}$ Eq. (7) $E_{\mathrm{IAS}} \approx 20.9-5.7 \Delta R_{\mathrm{np}}$ which is in reasonable agreement with the result of the linear fit in Fig. 1 , which gives $E_{\mathrm{IAS}} \approx 19.9(8)-5.0(2) \Delta R_{\mathrm{np}}$ and a linear correlation coefficient $r=-0.985$.

In Fig. 1, it is also shown that the model predictions are not compatible with our present knowledge on the studied observables. The experimental IAS energy [31] is shown (horizontal dashed line) in the figure, and a simple extrapolation implies $\Delta R_{\mathrm{np}}=0.07(2) \mathrm{fm}$. This value is incompatible with previous studies $[9,11,32]$. As a reference, recent experimental constraints from polarized proton elastic scattering [29], parity violating elastic electron scattering [19], and electric dipole polarizability [30], are indicated in the bottom part of Fig. 1.

To solve this puzzle, we have reconsidered in Ref. [1] all possible contributions to the IAS energy that have not been considered with sufficient care in self-consistent calculations. Those effects are briefly discussed in what follows and have been implemented within a Skyrme functional. Note that none of the new terms impacts to the proton-neutron RPA residual force.

\section{Electromagnetic spin-orbit}

The electromagnetic spin-orbit correction to the nucleon single-particle (sp) energy can be written within a nonrelativistic approximation as follows:

$$
\Delta \varepsilon_{i}=\frac{\hbar^{2} c^{2}}{2 m^{2} c^{4}} x_{i}\left\langle\vec{l}_{i} \cdot \vec{s}_{i}\right\rangle \int \frac{d r}{r} \frac{d U_{\mathrm{Coul}}}{d r} u_{i}^{2}(r),
$$

where $i$ labels the $i-$ th nucleon quantum numbers, $m_{i}$ is the neutron or proton mass, $U_{\mathrm{C}}$ is the Coulomb energy potential - calculated at the HF level or tree level - and $x_{i}$ is equal to $g_{p}-1$ for protons and $g_{n}$ for neutrons; $g_{n}=-3.82608545(90)$ and $g_{p}=5.585694702(17)$ are the neutron and proton $g$-factors, respectively [33]. The total 
energy correction to the binding energy of a nucleus will be then $\Delta E_{\text {emso }}=\sum_{i=1}^{A} \varepsilon_{i}^{\text {emso }}$. The effect of this term can be treated perturbatively since it produces a change in the nucleon single particle energies of tens of $\mathrm{keV}$ and, thus, negligible in the single particle wave functions.

As an exercise, assuming a uniformly charged sphere of radius $R_{\mathrm{C}}$ one can estimate the energy correction to the single particle energies [34]

$$
\varepsilon_{i}^{\mathrm{emso}} \approx-\frac{\hbar^{3} c^{3}}{2 m_{i}^{2} c^{4}} \frac{Z \alpha}{R_{\mathrm{C}}^{3}}\left\langle\vec{l}_{i} \cdot \vec{s}_{i}\right\rangle x_{i} .
$$

\section{Coulomb exchange}

In connection with the Skyrme interaction, it is customary to adopt the so-called Slater approximation for the calculation of the Coulomb exchange. The Slater approximation is local and, hence, it does not break the assumption of zero-range; thus, it allows the use of simpler codes when working in coordinate space. The direct part reads

$$
U_{\mathrm{C}}^{\mathrm{dir} .}(\vec{r}) \varphi_{i}(\vec{r})=\frac{e^{2}}{2} \int d^{3} r^{\prime} \frac{\rho_{p}\left(\vec{r}^{\prime}\right)}{\left|\vec{r}-\vec{r}^{\prime}\right|} \varphi_{i}(\vec{r}),
$$

while the exchange part within the Slater approximation is

$$
U_{\mathrm{C}}^{\mathrm{x}, \text { Slater }} \varphi_{i}(\vec{r})=-\frac{e^{2}}{2}\left(\frac{3}{\pi}\right)^{1 / 3} \rho_{p}^{1 / 3} \varphi_{i}(\vec{r}),
$$

where $e$ is the elementary electric charge, $\rho_{p}$ is the proton density and $\varphi_{i}$ is the single particle wave function of the $i-$ th nucleon. The exact exchange has instead the wellknown non-local form

$$
U_{\mathrm{C}}^{\mathrm{x}, \text { exact }} \varphi_{i}(\vec{r})=-\frac{e^{2}}{2} \int d^{3} r^{\prime} \frac{\varphi_{j}^{*}\left(\vec{r}^{\prime}\right) \varphi_{j}(\vec{r})}{\left|\vec{r}-\vec{r}^{\prime}\right|} \varphi_{i}\left(\vec{r}^{\prime}\right) .
$$

Since the early pioneering calculations done with the Skyrme force, the Slater approximation has been known to work quite well. In particular, the relative difference between the exact total energy and that calculated with the Slater approximation has been found to be of the order of $3-6 \%$ in Ref. [35]. Thus, we may expect that the effects arising from the replacement of the Slater approximation by the exact exchange can be treated in a perturbative prescription described below. Our strategy is that we replace the single particle energies with those calculated with exact Coulomb matrix elements, but do not change the HF wave functions calculated by the Slater approximation. While the Coulomb interaction contributes to the $\mathrm{HF}$ field, the Coulomb residual interaction have no contribution to charge-exchange RPA matrix elements.

In practice, the HF equation has been firstly solved within the Slater approximation

$$
\left[H+U_{\mathrm{C}}^{\mathrm{dir} .}+U_{\mathrm{C}}^{\mathrm{x}, \text { Slater }}\right] \varphi_{i}=\varepsilon_{i} \varphi_{i}
$$

Then, in a second step, the single-particle energies $\varepsilon_{i}$ have been corrected perturbatively, namely new energies $\tilde{\varepsilon}_{i}$ have been extracted from

$$
\tilde{\varepsilon}_{i}=\varepsilon_{i}+\int d^{3} r \varphi_{i}(\vec{r})^{*}\left(U_{\mathrm{C}}^{\mathrm{x}, \text { exact }}-U_{\mathrm{C}}^{\mathrm{x}, \text { Slater }}\right) \varphi_{i}(\vec{r}) .
$$

The single-particle energies undergo small changes due to the correction $\Delta U \equiv U_{\mathrm{C}}^{\mathrm{x} \text {,exact }}-U_{\mathrm{C}}^{\mathrm{x}, \text { Slater }}$, that is hundreds of $\mathrm{keV}$ at most (see for example [25]). Thus, considering the wave functions as unchanged is a fairly good assumption. We, therefore, will perform RPA with exact Coulomb exchange by just replacing proton single-particle energies $\varepsilon_{i}$ with their new values $\tilde{\varepsilon}_{i}$.

\section{Vacuum polarization correction}

The lowest order correction in the fine-structure constant to the Coulomb potential $e Z / r$ is estimated via the vacuum polarization correction. The vacuum polarization modifies the charge of the proton over a range of the order of the electron Compton wavelength. The virtual emission and absorption of an electron-positron pair gives rise to a repulsive potential $V_{\mathrm{vp}}$ that corrects the Coulomb repulsion of two protons. Taking into account the finite size of the nucleus, the correction potential can be written as follows: [36]:

$$
V_{\mathrm{vp}}(\vec{r})=\frac{2}{3} \frac{\alpha e^{2}}{\pi} \int d \vec{r}^{\prime} \frac{\rho\left(\vec{r}^{\prime}\right)}{\left|\vec{r}-\vec{r}^{\prime}\right|} \mathcal{K}_{1}\left(\frac{2}{\lambda_{e}}\left|\vec{r}-\vec{r}^{\prime}\right|\right),
$$

where $e$ is the fundamental electric charge, $\alpha$ the finestructure constant, $\lambda_{e}$ the reduced Compton electron wavelength and

$$
\mathcal{K}_{1}(x) \equiv \int_{1}^{\infty} d t e^{-x t}\left(\frac{1}{t^{2}}+\frac{1}{2 t^{4}}\right) \sqrt{t^{2}-1}
$$

\section{Finite size effects}

The electric charge distribution is calculated and consistently used in the HF calculation of the Coulomb potential by taking into account the effect of the neutron and proton electromagnetic form factors and the electromagnetic spin-orbit. Two approaches are used that give rise to the same root mean square charge radius of the nucleus under study. The first approach consist in doing the actual calculation with realistic proton and neutron electromagnetic form factors convoluted by using the proton and neutron point like densities, respectively. That is, up to order $1 / \mathrm{m}^{2}$ and assuming spherical symmetry [37]

$$
\begin{gathered}
\rho_{\mathrm{ch}}(q)=\left(1-\frac{q^{2}}{8 m^{2}}\right)\left[G_{\mathrm{E}, \mathrm{p}}\left(q^{2}\right) \rho_{p}(q)+G_{\mathrm{E}, \mathrm{n}}\left(q^{2}\right) \rho_{n}(q)\right] \\
-\frac{\pi q^{2}}{2 m^{2}} \sum_{l, t}\left[2 G_{\mathrm{M}, \mathrm{t}}\left(q^{2}\right)-G_{\mathrm{E}, \mathrm{t}}\left(q^{2}\right)\right]\langle\vec{l} \cdot \vec{s}\rangle \times \\
\int_{0}^{\infty} d x \frac{j_{1}(q x)}{q x}\left|R_{n, l, j}(x) x^{2}\right|^{2},
\end{gathered}
$$

where $G_{\mathrm{E}, \mathrm{M}}$ is taken from [38].

The second approach is phenomenological and takes into account -on average- the previously mentioned contributions by convoluting the proton point like density with a gaussian form factor with a conveniently chosen range

$$
\rho_{\mathrm{ch}}(q)=G\left(q^{2}\right) \rho_{p}(q)
$$

where the $G\left(q^{2}\right)$ takes a gaussian form with range $\mu=$ $0.65 \mathrm{fm}$. 


\section{Charge symmetry breaking and charge independence breaking potentials}

A charge symmetry breaking potential can be generally defined as $V_{\mathrm{CSB}}=V_{n n}-V_{p p}$ while a charge independence breaking potential can be generally defined as $V_{\mathrm{CIB}}=$ $\frac{1}{2}\left(V_{n n}+V_{p p}\right)-V_{p n}$. So an effective nucleon-nucleon potential can be build on three parts: the charge independent part (isospin symmetry is fully preserved) plus the two previously defined breaking terms.

Following [39] [cf. Eqs. (18)-(21)] one can define Skyrme-like CSB and CIB potentials as follows:

$$
\begin{aligned}
V_{\mathrm{CSB}}\left(\vec{r}_{1}, \vec{r}_{2}\right) \equiv \frac{1}{4}\left[\tau_{z}(1)+\tau_{z}(2)\right]\left\{s_{0}\left(1+y_{0} P_{\sigma}\right)\right. \\
+\frac{1}{2} s_{1}\left(1+y_{1} P_{\sigma}\right) \times\left[P^{\prime 2} \delta\left(\vec{r}_{1}-\vec{r}_{2}\right)+\delta\left(\vec{r}_{1}-\vec{r}_{2}\right) P^{2}\right] \\
\left.+s_{2}\left(1+y_{2} P_{\sigma}\right) \vec{P}^{\prime} \cdot \delta\left(\vec{r}_{1}-\vec{r}_{2}\right) \vec{P}\right\}
\end{aligned}
$$

and

$$
\begin{gathered}
V_{\mathrm{CIB}}\left(\vec{r}_{1}, \vec{r}_{2}\right) \equiv \frac{1}{2} \tau_{z}(1) \tau_{z}(2)\left\{u_{0}\left(1+z_{0} P_{\sigma}\right)\right. \\
+\frac{1}{2} u_{1}\left(1+z_{1} P_{\sigma}\right) \times\left[P^{\prime 2} \delta\left(\vec{r}_{1}-\vec{r}_{2}\right)+\delta\left(\vec{r}_{1}-\vec{r}_{2}\right) P^{2}\right] \\
\left.+u_{2}\left(1+z_{2} P_{\sigma}\right) \vec{P}^{\prime} \cdot \delta\left(\vec{r}_{1}-\vec{r}_{2}\right) \vec{P}\right\},
\end{gathered}
$$

where $\vec{P} \equiv \frac{1}{2 i}\left(\vec{\nabla}_{1}-\vec{\nabla}_{2}\right)$ acts on the right and $P^{\prime}$ is its complex conjugate acting on the left and $P_{\tau / \sigma}$ are the usual projector operators in isospin and spin spaces.

The Hartree-Fock energy density associated to the CSB and CIB potentials is

$$
\begin{aligned}
& \mathcal{H}_{\mathrm{CSB}}=\frac{s_{0}\left(1-y_{0}\right)}{8}\left(\rho_{n}^{2}-\rho_{p}^{2}\right) \\
& +\frac{s_{1}\left(1-y_{1}\right)}{32}\left[-\frac{3}{2} \rho_{n} \nabla^{2} \rho_{n}+\frac{3}{2} \rho_{p} \nabla^{2} \rho_{p}\right. \\
& \left.+2\left(\rho_{n} \tau_{n}-\rho_{p} \tau_{p}\right)+\left(\vec{J}_{n}^{2}-\vec{J}_{p}^{2}\right)\right] \\
& +\frac{3}{32} s_{2}\left(1+y_{2}\right)\left[\frac{1}{2}\left(\rho_{n} \nabla^{2} \rho_{n}-\rho_{p} \nabla^{2} \rho_{p}\right)\right. \\
& \left.+2\left(\tau_{n} \rho_{n}-\tau_{p} \rho_{p}\right)-\frac{1}{3}\left(\vec{J}_{n}^{2}-\vec{J}_{p}^{2}\right)\right] \text {. } \\
& \mathcal{H}_{\mathrm{CIB}}=\frac{1}{8}\left[u_{0}\left(1-z_{0}\right)\left(\rho_{n}^{2}+\rho_{p}^{2}\right)-2 u_{0}\left(2+z_{0}\right) \rho_{n} \rho_{p}\right] \\
& +\frac{1}{32}\left\{-\frac{3}{2} u_{1}\left(1-z_{1}\right)\left(\rho_{n} \nabla^{2} \rho_{n}+\rho_{p} \nabla^{2} \rho_{p}\right)\right. \\
& +\frac{3}{2} u_{1}\left(2+z_{1}\right)\left(\rho_{n} \nabla^{2} \rho_{p}+\rho_{p} \nabla^{2} \rho_{n}\right) \\
& +2 u_{1}\left(1-z_{1}\right)\left(\tau_{n} \rho_{n}+\tau_{p} \rho_{p}\right) \\
& -2 u_{1}\left(2+z_{1}\right)\left(\tau_{n} \rho_{p}+\tau_{p} \rho_{n}\right) \\
& \left.+u_{1}\left(1-z_{1}\right)\left(\vec{J}_{n}^{2}+\vec{J}_{p}^{2}\right)+2 u_{1} z_{1} \vec{J}_{n} \vec{J}_{p}\right\}
\end{aligned}
$$

Table 1. Effect of the different contributions from isospin breaking (including both CSB and CIB) mentioned in the text on the IAS energy in ${ }^{208} \mathrm{~Pb}$. Corrections are basically model-independent except the last one.

\begin{tabular}{lrr}
\hline & $E_{\mathrm{IAS}}[\mathrm{MeV}]$ & Corr. $[\mathrm{keV}]$ \\
\hline No corrections & 18.31 & \\
Exact Coulomb exchange & 18.41 & +100 \\
n/p mass difference & 18.44 & +30 \\
Electromagnetic spin-orbit & 18.45 & +10 \\
Finite size effects & 18.40 & -50 \\
Vacuum polarization $\left(V_{\text {ch }}\right)$ & 18.53 & +130 \\
\hline Isospin symmetry breaking & 18.80 & +270 \\
\hline
\end{tabular}

$$
\begin{aligned}
+\frac{1}{16}\left[\frac{3}{4} u_{2}\left(1+z_{2}\right)\left(\rho_{n} \nabla^{2} \rho_{n}+\rho_{p} \nabla^{2} \rho_{p}\right)\right. \\
-\frac{1}{4} u_{2}\left(2+z_{2}\right)\left(\rho_{p} \nabla^{2} \rho_{n}+\rho_{p} \nabla^{2} \rho_{n}\right) \\
+3 u_{2}\left(1+z_{2}\right)\left(\tau_{n} \rho_{n}+\tau_{p} \rho_{p}\right) \\
-u_{2}\left(2+z_{2}\right)\left(\tau_{n} \rho_{p}+\tau_{p} \rho_{n}\right) \\
\left.-\frac{1}{2} u_{2}\left(1+z_{2}\right)\left(\vec{J}_{n}^{2}+\vec{J}_{p}^{2}\right)+u_{2} z_{2} \vec{J}_{n} \vec{J}_{p}\right]
\end{aligned}
$$

\section{Results}

The contributions in Sec. 2-5, produce an overall - model independent - upward shift of the IAS energy for the straight line that connects the points of Fig. 1. In Table 1, we can see that this shift, by adding also the small effect of the neutron-proton mass difference, amounts to $\approx 220 \mathrm{keV}$. This is too small in order to let the line intersect the experimental value for the IAS energy at a point that corresponds to a realistic range of $\Delta R_{\mathrm{np}}$ (indicated by the horizontal bars in Fig. 1).

CSB and CIB effects have been widely discussed in the literature (see, for example, Refs. [40-42]). Recently, the isospin mixing in ${ }^{8} \mathrm{Be}$ was studied based on the Green's function Monte Carlo method by including the CSB interaction [43]. Although it is known for many years that CSB-CIB forces must be taken into account to reproduce the so-called Nolen-Schiffer anomaly along the nuclear chart, the information on CSB-CIB forces in the nuclear medium is scarce. The nuclear shell model has been employed for quite some time to analyze the energies along the isobaric multiplets [34]. In the same context, it has been noticed that CSB-CIB interactions needed to explain the data are not consistent with those in the vacuum [44]. Similar conclusions have been drawn in Ref. [45].

In the present work we have kept our description simple. We considered only the terms in $s_{0}$ and $u_{0}$ (see Sec. 6) to be different from zero with $y_{0}$ and $z_{0}$ fixed to -1 . The momentum-dependent terms have not been considered, under the rationale that the information that we have at our disposal is not sufficient to pin down the val- 
Table 2. SAMi-ISB parameter set. The statistical errors $\sigma$ are given in parenthesis. See text for details.

\begin{tabular}{lrl}
\hline & value $(\sigma)$ & \\
\hline$t_{0}$ & $-2098.3(149.3)$ & $\mathrm{MeV} \mathrm{fm}^{3}$ \\
$t_{1}$ & $394.7(15.8)$ & $\mathrm{MeV} \mathrm{fm}^{5}$ \\
$t_{2}$ & $-136.4(10.8)$ & $\mathrm{MeV} \mathrm{fm}^{5}$ \\
$t_{3}$ & $11995(686)$ & $\mathrm{MeV} \mathrm{fm}^{3+3 \alpha}$ \\
$x_{0}$ & $0.242(9)$ & \\
$x_{1}$ & $-0.17(33)$ & \\
$x_{2}$ & $-0.470(4)$ & \\
$x_{3}$ & $0.32(21)$ & \\
$W_{0}$ & $294(6)$ & \\
$W_{0}^{\prime}$ & $-367(12)$ & \\
$\alpha$ & $0.223(31)$ & \\
$s_{0}$ & $-26.3(7)$ & $\mathrm{MeV} \mathrm{fm}^{3}$ \\
$u_{0}$ & $25.8(4)$ & $\mathrm{MeV} \mathrm{fm}^{3}$ \\
\hline
\end{tabular}

ues of all parameters of a general interaction with several partial waves.

In Ref. [46], ISB contributions to the energy per particle of symmetric nuclear matter have been studied within the Brueckner-Hartree-Fock theory based on AV18 realistic potential [47]. We have determined a new Skyrme parameter set named SAMi-ISB, using the same fitting protocol of SAMi but including CSB-CIB contributions (see Ref. [1] and supplemental material). We have first started from existing parameters of SAMi and fixed the values of $s_{0}$ and $u_{0}$ by requiring a reproduction of the results of Ref. [46] and the value of the IAS energy in ${ }^{208} \mathrm{~Pb}$. Then, the standard Skyrme parameters have been refitted to ensure the realiability of the isospin conserving part of the Skyrme functional. This has been done in two steps since $\mathrm{CSB} / \mathrm{CIB}$ affect the binding energies and charge radii only by a few per mil or per cent.

The values of the SAMi-ISB parameters are provided in Table 2. As seen in Fig. 1, with SAMi-ISB, the IAS energy of ${ }^{208} \mathrm{~Pb}$ is predicted at $E_{\mathrm{IAS}}=18.80(5) \mathrm{MeV}$ $\left(E_{\mathrm{IAS}}^{\exp }=18.826 \pm 0.010 \mathrm{MeV}\right.$ [31] $)$ with the neutron skin $\Delta R_{\mathrm{np}}=0.151(7) \mathrm{fm}$, which is within the realistic range deduced from the three experiments. Moreover, we have checked the predictive power of SAMi-ISB by calculating the IAS energy in other nuclei (see supplemental material in Ref. [1]).

In conclusion, SAMi-ISB is a new parameterization of a Skyrme-like energy density functional (EDF) that reconcile standard nuclear properties (saturation density, binding energy and charge radii of finite nuclei) with both our current understanding of the density behavior of the symmetry energy and the reproduction of the IAS energy of ${ }^{208} \mathrm{~Pb}$. To achieve that, we have self-consistently included for the first time within the HF+RPA framework all known contributions that break the isospin symmetry. All of these contributions are calculated in a model-independent way, except the CSB-CIB contribution. We have fixed only two free parameters in the CSB-CIB terms, and we have shown that this allows reproducing at the same time BHF calculations based on AV18, and the IAS energy of a heavy nucleus such as ${ }^{208} \mathrm{~Pb}$ without compromising the other properties of nuclear matter and finite nuclei.
Funding from the European Union's Horizon 2020 research and innovation programme under grant agreement No 654002 is acknowledged.

\section{References}

[1] X. Roca-Maza, G. Colò, H. Sagawa, Phys. Rev. Lett. 120, 202501 (2018)

[2] M. Oertel, M. Hempel, T. Klähn, S. Typel, Rev. Mod. Phys. 89, 015007 (2017)

[3] X. Roca-Maza, N. Paar, Prog. Part. Nucl. Phys. 101, 96 (2018)

[4] B.A. Li, A. Ramos, G. Verde, I. Vidaña, Eds., Eur. Phys. J. A 50 (2014)

[5] M. Baldo, G.F. Burgio, Prog. Part. Nucl. Phys. 91, 203 (2016)

[6] M. Tsang, Z. Chajecki, D. Coupland, P. Danielewicz, F. Famiano, R. Hodges, M. Kilburn, F. Lu, W. Lynch, J. Winkelbauer et al., Prog. Part. Nucl. Phys. 66, 400 (2011)

[7] J. Carriere, C.J. Horowitz, J. Piekarewicz, Astrophys. J. 593, 463 (2003)

[8] T. Sil, M. Centelles, X. Viñas, J. Piekarewicz, Phys. Rev. C 71, 045502 (2005)

[9] J.M. Lattimer, Y. Lim, Astrophys. J. 771, 51 (2013)

[10] B.A. Li, X. Han, Phys. Lett. B 727, 276 (2013)

[11] C.J. Horowitz, E.F. Brown, Y. Kim, W.G. Lynch, R. Michaels, A. Ono, J. Piekarewicz, M.B. Tsang, H.H. Wolter, J. Phys. G: Nucl. Part. Phys. 41, 093001 (2014)

[12] B. Alex Brown, Phys. Rev. Lett. 85, 5296 (2000)

[13] S. Typel, B.A. Brown, Phys. Rev. C 64, 027302 (2001)

[14] R. Furnstahl, Nucl. Phys. A 706, 85 (2002)

[15] M. Centelles, X. Roca-Maza, X. Viñas, M. Warda, Phys. Rev. Lett. 102, 122502 (2009)

[16] X. Roca-Maza, M. Centelles, X. Viñas, M. Warda, Phys. Rev. Lett. 106, 252501 (2011)

[17] B.A. Brown, Phys. Rev. Lett. 119, 122502 (2017)

[18] S. Abrahamyan et al. (PREX Collaboration), Phys. Rev. Lett. 108, 112502 (2012)

[19] C.J. Horowitz, Z. Ahmed, C.M. Jen, A. Rakhman, P.A. Souder, M.M. Dalton, N. Liyanage, K.D. Paschke, K. Saenboonruang, et al., Phys. Rev. C 85, 032501 (2012)

[20] C.J. Horowitz, K.S. Kumar, R. Michaels, Eur. Phys. J. A 50, 48 (2014)

[21] M. Bender, P.H. Heenen, P.G. Reinhard, Rev. Mod. Phys. 75, 121 (2003)

[22] G. Colò, N. Van Giai, P.F. Bortignon, R.A. Broglia, Phys. Rev. C 50, 1496 (1994)

[23] N. Paar, T. Nikšić, D. Vretenar, P. Ring, Phys. Rev. C 69, 054303 (2004)

[24] X. Roca-Maza, G. Colò, H. Sagawa, Phys. Rev. C 86, 031306 (2012)

[25] X. Roca-Maza, L.G. Cao, G. Colò, H. Sagawa, Phys. Rev. C 94, 044313 (2016) 
[26] X. Roca-Maza, M. Brenna, B.K. Agrawal, P.F. Bortignon, G. Colò, L.G. Cao, N. Paar, D. Vretenar, Phys. Rev. C 87, 034301 (2013)

[27] D. Vretenar, T. Nikšić, P. Ring, Phys. Rev. C 68, 024310 (2003)

[28] N. Auerbach, J. Hüfner, A.K. Kerman, C.M. Shakin, Phys. Rev. Lett. 23, 484 (1969)

[29] J. Zenihiro, H. Sakaguchi, T. Murakami, M. Yosoi, Y. Yasuda, S. Terashima, Y. Iwao, H. Takeda, M. Itoh, et al., Phys. Rev. C 82, 044611 (2010)

[30] X. Roca-Maza, X. Viñas, M. Centelles, B.K. Agrawal, G. Colò, N. Paar, J. Piekarewicz, D. Vretenar, Phys. Rev. C 92, 064304 (2015)

[31] M. Martin, Nuclear Data Sheets 108, 1583 (2007)

[32] X. Viñas, M. Centelles, X. Roca-Maza, M. Warda, Eur. Phys. J. A 50, 27 (2014)

[33] P.J. Mohr, D.B. Newell, B.N. Taylor, Rev. Mod. Phys. 88, 035009 (2016)

[34] M. Bentley, S. Lenzi, Prog. Part. Nucl. Phys. 59, 497 (2007)

[35] C. Titin-Schnaider, P. Quentin, Phys. Lett. B 49, 397 (1974)

[36] L.W. Fullerton, G.A. Rinker, Phys. Rev. A 13, 1283 (1976)
[37] L. Ray, Phys. Rev. C 19, 1855 (1979)

[38] J. Friedrich, T. Walcher, Eur. Phys. J. A 17, 607 (2003)

[39] H. Sagawa, N.V. Giai, T. Suzuki, Phys. Lett. B 353, 7 (1995)

[40] E.M. Henley, G.A. Miller, in Mesons in Nuclei, edited by M. Rho, D.H. Wilkinson (North-Holland, Amsterdam, 1979)

[41] G.A. Miller, B.M.K. Nefkens, I. Slaus, Phys. Rep. 194, 1 (1990)

[42] U. van Klock, J.L. Friar, T. Goldmann, Phys. Lett. B 371, 169 (1996)

[43] R.B. Wiringa, S. Pastore, C. Pieper, G.A. Miller, Phys. Rev. C 88, 044333 (2013)

[44] M.A. Bentley, S.M. Lenzi, S.A. Simpson, C.A. Diget, Phys. Rev. C 92, 024310 (2015)

[45] P. Baczyk, J. Dobaczewski, M. Konieczka, W. Satula, T. Nakatsukasa, K. Sato, Phys. Lett. B 778, 178 (2018)

[46] H. Muther, A. Polls, R. Machleidt, Phys. Lett. B 445, 259 (1999)

[47] R.B. Wiringa, V.G.J. Stoks, R. Schiavilla, Phys. Rev. C 51, 38 (1995) 